\title{
Clinical evaluation of intensity-modulated radiotherapy for locally advanced pancreatic cancer
}

\author{
Yoko Goto ${ }^{1 *}$ D , Akira Nakamura', Ryo Ashida', Katsuyuki Sakanaka', Satoshi Itasaka', Keiko Shibuya², \\ Shigemi Matsumoto ${ }^{3}$, Masashi Kanai ${ }^{3}$, Hiroyoshi Isoda ${ }^{4}$, Toshihiko Masui ${ }^{5}$, Yuzo Kodama ${ }^{6}$, Kyoichi Takaori ${ }^{5}$, \\ Masahiro Hiraoka ${ }^{7}$ and Takashi Mizowaki ${ }^{1}$
}

\begin{abstract}
Background: The purpose was to retrospectively evaluate the effect of intensity-modulated radiotherapy (IMRT) on gastrointestinal (Gl) toxicities and outcomes compared to three-dimensional conformal radiotherapy (3DCRT) for locally advanced pancreatic cancer (LAPC).

Methods: We included 107 consecutive patients who underwent CRT for LAPC from September 2001 to March 2015; 80 patients underwent 3DCRT and 27 patients underwent IMRT. They were compared for GI toxicities, locoregional progression free survival (LRPFS), distant metastasis free survival (DMS), and overall survival (OS).

Results: Median radiation dose and fractions for 3DCRT and IMRT were $54 \mathrm{~Gy} / 30 \mathrm{fr}$. and $48 \mathrm{~Gy} / 15 \mathrm{fr}$. The regimens of CRT consisted of weekly gemcitabine $250 \mathrm{mg} / \mathrm{m}^{2}$ (for 3DCRT) or $1000 \mathrm{mg} / \mathrm{m}^{2}$ (for IMRT). Acute Gl toxicity $\geq$ grade 2 occurred in 32 patients (40\%) treated with 3DCRT compared with five patients (19\%) treated with IMRT. Late Gl toxicity of grade 3 occurred in 10 patients (12\%) treated with 3DCRT and one patient (4\%) treated with IMRT. Patients who underwent IMRT had superior 1-year LRPFS (73.1\% vs. 63.2\%, $p=0.035)$ and 1 -year OS $(92.3 \%$ vs. $68.2 \%, p=0.037)$ as compared with those treated with 3DCRT. Multivariate analysis showed that in IMRT patients, higher dose ( $\geq 45$ Gy) was an independent factor for better LRPFS and OS.

Conclusions: LAPC patients treated with hypofractionated full-dose gemcitabine IMRT had improved OS and LRPFS without increased GI toxicities when compared to those of patients treated with conventionally fractionated low dose gemcitabine 3DCRT. In IMRT patients, higher dose was an independent favorable prognostic factor for better LRPFS and OS, which suggests that dose escalation with IMRT for LAPC is a promising strategy.
\end{abstract}

Keywords: Intensity-modulated radiotherapy, Chemoradiotherapy, Locally advanced pancreatic cancer, Treatment result

\section{Background}

Pancreatic cancer is fatal for most patients, and it is the fourth leading cause of death from malignancies in Japan. Only surgical resection offers a potentially curative approach, but merely 5 to $25 \%$ of patients present with resectable disease $[1,2]$. Approximately $35 \%$ of patients with pancreatic cancer have unresectable locally

\footnotetext{
* Correspondence: ygoto@kuhp.kyoto-u.ac.jp

${ }^{1}$ Department of Radiation Oncology and Image-applied Therapy, Kyoto University Graduate School of Medicine, 54 Shogoin, Kawaracho, Sakyo-ku, Kyoto, Japan

Full list of author information is available at the end of the article
}

advanced pancreatic cancer (LAPC), and the treatment for them is chemotherapy with or without radiotherapy [3]. The prognosis of LAPC patients has been poor, with a median survival of 9 to 13 months and 5-year overall survival (OS) of $<5 \%$. Distant metastases are a dominant cause of disease progression. However, a recent autopsy study revealed that about one-third of patients with pancreatic cancer die from locally destructive disease rather than distant metastasis [4]. This suggests that local control is meaningful to prevent tumor progression and improved survival of LAPC patients.

(C) The Author(s). 2018 Open Access This article is distributed under the terms of the Creative Commons Attribution 4.0 International License (http://creativecommons.org/licenses/by/4.0/), which permits unrestricted use, distribution, and 
For several decades, the role of radiotherapy for LAPC remained controversial. Since the 1980s, several randomized control trials of LAPC patients comparing chemoradiotherapy (CRT) with chemotherapy were conducted [5-7]. However, the results were contradictory. The results of a recently published randomized LAP07 trial demonstrated no significant survival benefits with the addition of radiotherapy to chemotherapy for LAPC patients [8]. In addition, more intensive regimens of chemotherapy, such as FOLFIRINOX and gemcitabine plus nab-paclitaxel, are reported to improve survival of metastatic pancreatic cancer patients, and are expected to be effective for LAPC patients [9-11]. This raises serious questions about the role of radiotherapy in the management of LAPC patients.

The biggest problem with CRT for pancreatic cancer is that tumor is surrounded by radiosensitive gastrointestinal (GI) organs, such as the stomach and the duodenum. This anatomical situation makes it difficult to deliver high doses to tumor without increasing irradiation dose to GI organs. The severe GI toxicity is related with the irradiated volume and dose received on the stomach and duodenum [12]. With the introduction of intensity-modulated radiotherapy (IMRT) which can simultaneously reduce the dose to surrounding normal organs, while allowing an increase in target tumor dose, it is expected that the rate of GI toxicities will be reduced and the efficacy may be increased [13, 14].

In our institution, we have been performing IMRT for LAPC since 2009. Before IMRT, we treated LAPC patients with 3DCRT at a total dose of $54 \mathrm{~Gy} / 30 \mathrm{fr}$. with weekly gemcitabine at $250 \mathrm{mg} / \mathrm{m}^{2}$ based on the results of phase I and phase II trials in our institution $[15,16]$. The treatment regimen was well-tolerated and provided prolonged survival in LAPC patients; the 1-year survival rate was $74 \%$ and the MST was 16.6 months. However, the rate of distant metastasis was high. When we introduced IMRT for LAPC, we decided to use full dose gemcitabine in combination with radiotherapy and shorten radiation fractionation from thirty to fifteen, based on a previous report [17]. We conducted phase I dose escalation study to determine the maximum tolerated radiation dose delivered by IMRT with full-dose gemcitabine, and it was $48 \mathrm{~Gy} / 15$ fr., (biological equivalent dose $\left(\mathrm{BED}_{10}\right)=63.4 \mathrm{~Gy}$, UMIN000004589). In this study, our aim is to retrospectively evaluate the effect of IMRT on outcomes and treatment-related acute and late GI toxicities compared with 3DCRT for LAPC.

\section{Methods}

\section{Patient characteristics}

The clinical data of LAPC patients treated with definitive CRT from September 2001 to March 2015 at our institution were retrospectively reviewed. Locally advanced unresectable disease was defined as superior mesenteric artery or celiac axis encasement $>180$ degrees, unreconstructible superior mesenteric vein/portal occlusion, or aortic invasion. Patient, tumor, and treatment characteristics were obtained from the medical records.

All patients were monitored weekly during CRT for acute GI toxicities, including nausea, vomiting, diarrhea, and abdominal pain. After CRT ended, patients were followed up once every 2 months, and monitored for late GI toxicities, including gastroduodenal ulcer and hemorrhage. All toxicities were scored according to the Common Terminology Criteria for Adverse Events (CTCAE), version 4.0. The institutional Review Board of Kyoto University Hospital approved this study.

\section{Chemotherapy}

The regimen of induction chemotherapy consisted of weekly intravenous administration of $1000 \mathrm{mg} / \mathrm{m}^{2}$ of gemcitabine on days $1,8,15$ during 4 -weeks. The regimens of CRT consisted of weekly gemcitabine $250 \mathrm{mg} /$ $\mathrm{m}^{2}$ (for 3DCRT) or $1000 \mathrm{mg} / \mathrm{m}^{2}$ (for IMRT). As additional treatment after radiotherapy, 3 weekly doses of gemcitabine at $1000 \mathrm{mg} / \mathrm{m}^{2}$ every 28 days were administered until the tumor progression or patient refusal. If patients had some complications associated with gemcitabine, such as intestinal pneumonia, $80 \mathrm{mg} / \mathrm{m}^{2} /$ day of S-1 was administered orally during radiotherapy twice daily on weekdays. After first-line gemcitabine-based therapy, S-1 based chemotherapies are the most frequently used, although this was left to the discretion of the medical oncologist.

\section{Radiotherapy}

Treatment-planning computed tomography (CT) was performed with intravenous contrast media, without oral contrast agents. The gross target volume (GTV) included radiographically apparent gross tumor and suspicious/enlarged lymph nodes on CT simulation for 3DCRT plans. The clinical target volume (CTV) was defined as the GTV plus a 5-mm margin in all directions. The CTV also included the potential para-aortic lymph node and neuroplexus involvement between the celiac axis and the superior mesenteric artery. The planned target volume (PTV) was determined by adding a horizontal 5-mm margin and a cephalocaudal $10-\mathrm{mm}$ margin to the CTV, considering respiratory movement. A total dose of 54 Gy was delivered in 30 fractions, using 3- or 4-field planning and a dynamic arc conformal technique.

For IMRT plans, the GTV and CTV were the same as those of 3DCRT plans. The PTV was generated by adding a 5-mm margin in all directions to the CTV in consideration of respiratory management. For IMRT, breath-hold method or tumor dynamic tracking method were adopted for the management of tumor respiratory 
motion. IMRT planning was performed, using a commercially available planning system Eclipse $^{\mathrm{TM}}$ Varian, Medical Systems, Palo Alto, CA). The prescription dose was specified as D95 (the dose that covers $95 \%$ of the structure) to PTV-boost, a volume that subtracted normal organs (the stomach and the duodenum) plus 5- to $10-\mathrm{mm}$ margins from PTV. The prescription dose was individualized between 39 and $51 \mathrm{~Gy} / 15 \mathrm{fr}$. by achieving the dose constrain for OARs and referring to a previous institutional trial (UMIN000004589). The dose constraints of the OARs are listed in Table 1. The standard beam arrangement involved five to six gantry angles. Treatment was delivered using dynamic multileaf collimation.

\section{Statistics}

Fisher exact test was performed to compare the characteristics of patients treated with 3DCRT versus those treated with IMRT. Overall survival (OS) was defined as the period from the chemotherapy or chemoradiotherapy starting date to the date of death of any cause, and it was censored at the last follow-up visit for living patients. The Kaplan-Meier method was used to estimate the OS, LRPFS, and DMFS. The log-rank test was performed for the OS, LRPFS, and DMFS comparisons. The Cox proportional hazard model was used to estimate the hazard ratio. The chi-squared test was used to compare the rates of acute GI toxicities and the Gray's test was used to compare the cumulative incidence rates of late GI toxicities of patients treated with 3DCRT versus those with IMRT. All statistical tests were 2-sided. The difference was deemed statistically significant when the $p$ value was $<.05$. All statistical analyses were performed using EZR version 1.11 (Saitama Medical Center, Jichi Medical University, Saitama, Japan), which is a graphical user interface for $\mathrm{R}$ version 2.13.2 (The R Foundation for Statistical Computing, Vienna, Austria).

Table 1 Dose constraints for OAR

\begin{tabular}{|c|c|}
\hline Structure & Constraints \\
\hline \multirow[t]{3}{*}{ Stomach/Duodenum } & V45 Gy $<1$ cc \\
\hline & V42 Gy $<5$ cc \\
\hline & V39 Gy $<25$ cc \\
\hline \multirow[t]{2}{*}{ Stomach + PRV/Duodenum + PRV } & V39 Gy $<30 c c$ \\
\hline & V36 Gy $<45 c c$ \\
\hline Spinal cord & Dmax <36 Gy \\
\hline Spinal cord + PRV & D2 cc < 39 Gy \\
\hline Kidney (at least one) & V20 Gy $<30 \%$ \\
\hline Liver & Dmean < 30 Gy \\
\hline
\end{tabular}

\section{Results}

Patient and tumor characteristics

In total, 107 consecutive patients who underwent CRT for LAPC from September 2001 to March 2015 were included in this analysis; 80 patients were treated with 3DCRT (75\%), and 27 patients with IMRT (25\%). Of the 107 patients, 58\% were male, and the median age at diagnosis was 65 years old (range, 35-85 years old). Fifty-three percent of tumors were located in the head/ neck of the pancreas and $47 \%$ in the body/tail.

Sixty-three (59\%) patients received induction chemotherapy, administered for 1-12 months prior to radiotherapy. In our protocol of IMRT clinical trial, the induction chemotherapy is one course of weekly administration of gemcitabine on days 1, 8, 15 during 4-weeks. All patients received concurrent chemotherapy during radiotherapy. Most patients (93\%) received gemcitabine, 8 patients $(7 \%)$ received it with $\mathrm{S}-1$. Median radiation doses and fractions for 3DCRT and IMRT were $54 \mathrm{~Gy} /$ 30 fr. (range, 48.6-55.6 Gy) and 48 Gy/15 fr. (range, 3951 Gy), respectively. Patient, tumor, and treatment characteristics are summarized in Table 2.

\section{Treatment outcome}

Median follow-up period was 16.4 months, which was similar between groups. The 1-year OS rate of all patients was $74.3 \%$, and median survival time (MST) was 17.5 months. The 1-year OS rates in the 3DCRT and IMRT groups were 68.2 and $92.3 \%$, respectively ( $p=$ $0.0369)$. The 1 -year LRPFS rates in the 3DCRT and IMRT groups were 63.2 and $73.1 \%$, respectively ( $p=$ 0.0349). The 1-year DMFS rates in the 3DCRT and IMRT groups were 48.4 and $49.3 \%$, respectively ( $p=$ 0.308) (Fig. 1).

\section{Univariate and multivariate analyses in all patients}

The results of univariate analyses for LRPFS and OS are shown in Table 3. Radiation modality of 3DCRT ( $p=$ $0.037)$, larger tumor $(p=0.047)$, and high pretreatment CA19-9 level $(p=0.010)$ were found to be significant unfavorable factors for OS. Younger age $(p=0.039)$, radiation modality of 3DCRT $(p=0.035)$, and high pretreatment CA19-9 level $(p<0.01)$ were significant unfavorable factors for LRPFS.

The results of multivariate analysis for OS are listed in Table 4. Multivariate analyses revealed that high pretreatment CA19-9 level $(p=0.026)$ was a significant unfavorable factor and radiation modality of 3DCRT $(p=0.082)$ was a marginal unfavorable factor.

\section{Univariate and multivariate analyses in patients treated with IMRT}

The results of univariate analyses for LRPFS and OA are shown in Table 5. Radiation dose under 45Gy $(p<0.01)$ 
Table 2 Patient characteristics of tumors and treatment

\begin{tabular}{llll}
\hline Characteristic & 3DCRT & IMRT & p-value \\
\hline Number of patients ( $\mathrm{n})$ & 80 & 27 & $66,42-84$ \\
Age (median, range) & $65,35-85$ & $10 / 17$ & 0.57 \\
Gender (male/female) & $45 / 35$ & $27 / 0$ & 0.65 \\
PS (0-1/2) & $74 / 6$ & $16 / 10$ & 3.33 \\
Tumor location(head, uncus/body, tail) & $41 / 39$ & $30,10-70$ \\
Tumor size (median, range [mm]) & $29,19-40$ & $27 / 0$ & 0.51 \\
Induction chemotherapy (yes/no) & $36 / 44$ & $26 / 1$ & 0.038 \\
Concurrent chemotherapy (Gemcitabine/S-1) & $73 / 7$ & $48,39-51$ & 0.01 \\
Radiation dose (median, range [Gy]) & $54,48.6-55.8$ & $15,15-15$ \\
Radiation fractionation (median, range) & $30,27-31$ & & 0.68 \\
\hline
\end{tabular}

Abbreviations; PS performance status

and high pretreatment CA19-9 level $(\mathrm{p}=0.037)$ were found to be significant unfavorable factors for OS. Younger age $(p=0.015)$, radiation dose under 45Gy $(\mathrm{p}<0.01)$, and high pretreatment CA19-9 level $(p=0.032)$ were significant unfavorable factors for LRPFS. As younger age was a significant unfavorable factor, it would be important to observe younger patients closely post-CRT.

The results of multivariate analyses for LRPFS and OA are listed in Table 6. Multivariate analyses revealed that radiation dose under $45 \mathrm{~Gy}$ was a significant unfavorable factor for both LRPFS $(\mathrm{p}<0.01)$ and OS $(p=0.044)$. Eighteen patients in the IMRT group who were treated with $\geq 45$ Gy had significantly better 1-year LRPFS $(94.1 \%$ vs. $33.3 \%, \mathrm{p}<0.01)$ and 1 -year OS $(94.1 \%$ vs. $88.9 \%, \mathrm{p}<0.01)$ compared with IMRT patients treated with $<45$ Gy (Fig. 2). There was no significant difference of tumor sizes among IMRT patients who had been treated with under $45 \mathrm{~Gy}$ and over $45 \mathrm{~Gy}$ (31.4 mm vs. $27.2 \mathrm{~mm}, p=0.18$ ). The MST of patients treated with IMRT over $45 \mathrm{~Gy}$ and under $45 \mathrm{~Gy}$ were 28.8 months and 14.3 months, respectively.

\section{Recurrence pattern}

Among the 27 patients who underwent IMRT, the first relapse occurred at locoregional in 3 patients (11.1\%) and at distant organs in 16 patients (59.3\%), and at both locoregional and distant organs in 1 patient (3.7\%). Among the 80 patients who underwent 3DCRT, the first relapse occurred at locoregional in 24 patients (30\%) and at distant organs in 42 patients $(52.5 \%)$, and at both locoregional and distant organs in 4 patients (5\%).

\section{Gl toxicities}

An overview of the acute and late GI toxicities and their grades is shown in Table 7. Acute GI toxicity of grade 2 or higher occurred in 32 patients (40\%) treated with 3DCRT compared with five patients $(19 \%)$ treated with
IMRT $(p=0.042)$. Late GI toxicity of grade 2 or higher occurred in 10 patients $(12 \%)$ treated with 3DCRT compared with two patients (8\%) treated with IMRT. As for late GI toxicity of grade 2 or higher, grade 3 toxicities occurred in 10 patients (12\%) treated with 3DCRT and one patient (4\%) treated with IMRT, respectively. GI toxicity of grade 4 or higher was not observed. The 1-year cumulative incidence rates of late GI toxicities of grade 2 or higher in the 3DCRT and IMRT groups were 11.3\% $(95 \% \mathrm{CI}=5.5-19.4 \%)$ and $7.6 \%(95 \% \mathrm{CI}=1.3-21.7 \%)$, respectively $(p=0.47)$.

\section{Discussion}

The present study aimed to evaluate the effect of hypofractionated full-dose gemcitabine IMRT on GI toxicities and outcomes compared to conventionally fractionated low dose gemcitabine 3DCRT for LAPC patients.

Pancreas is surrounded by radiosensitive GI organs such as the stomach and the duodenum, and this anatomical situation makes it difficult to deliver high doses to tumor without increasing the irradiation dose to GI organs $^{12,13}$. A previous attempt for dose-escalation when performing 3DCRT resulted in failure in LAPC; it was more toxic and less effective [6]. Because IMRT can deliver high-dose radiation to the target volume while decreasing the radiation to dose-limiting adjacent critical structures, it has been suggested to be beneficial for LAPC. A systematic review showed that GI toxicities were significantly reduced with IMRT [18]. Similar to the previous reports, the rates of GI toxicities were low in our study; only $4 \%$ of IMRT patients experienced grade 3+ late GI toxicity, compared with $12 \%$ of 3DCRT patients. In addition, concerning acute hematological toxicities in the IMRT patients, grade 3 leukopenia, neutropenia and thrombocytopenia occurred in 10,7 , and 1 patients, respectively. Grade 4 

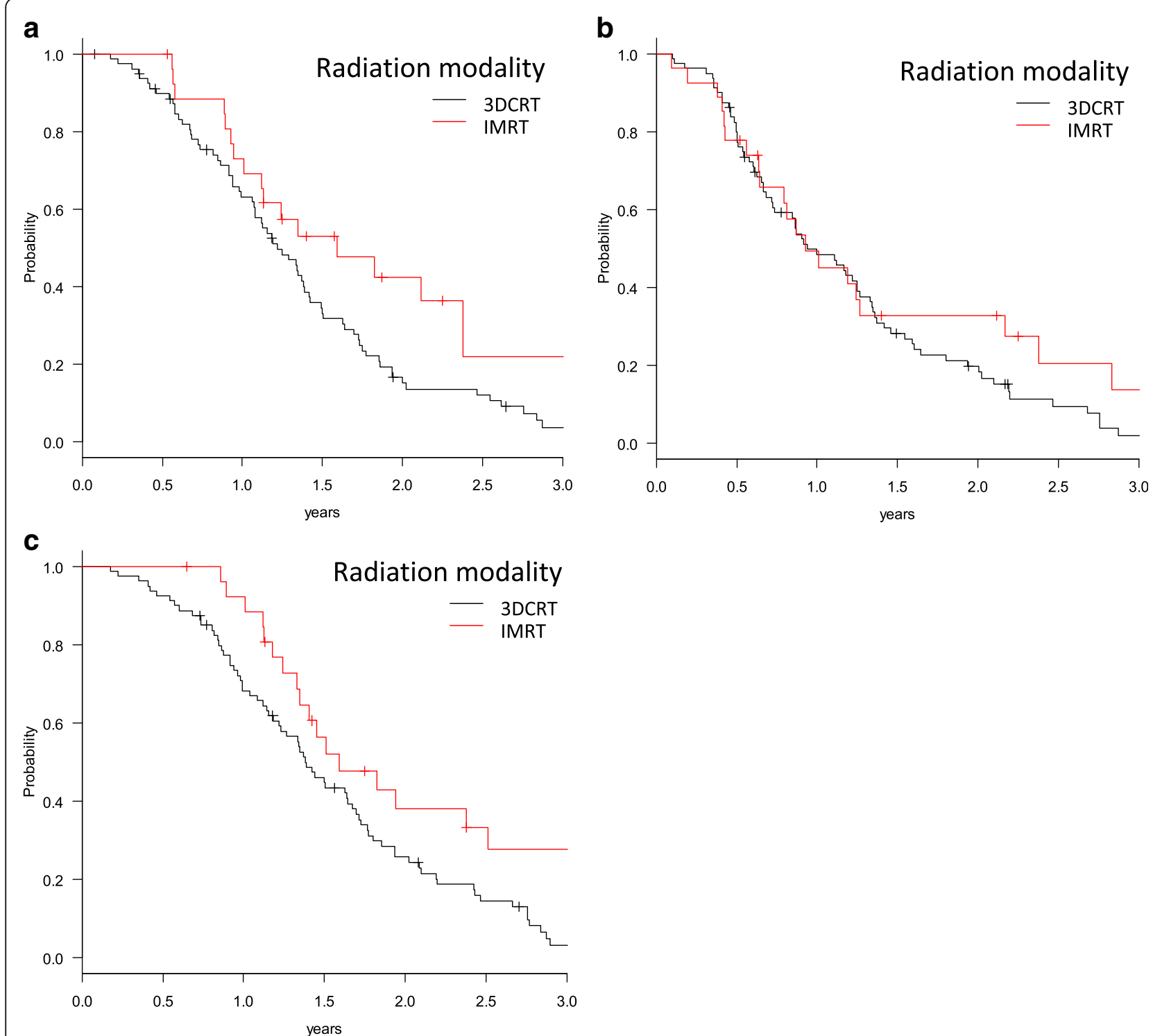

Fig. 1 Kaplan-Meier estimates of (a) Locoregional progression free survival (LRPFS), (b) Distant metastasis free survival (DMFS) and (c) overall survival (OS) by radiation modality of patients who received 3DCRT $(n=80)$ and IMRT $(n=27)$

neutropenia occurred in 3 patients (11\%); however, all acute hematologic toxicities were able to be well managed. IMRT enabled us to use full dose gemcitabine in combination with radiotherapy of shorten fractionation without increasing GI toxicities when compared to 3DCRT.

This study revealed that patients treated with IMRT showed significantly improved LRPFS and OS compared with those treated with 3DCRT, whereas there was no significant difference in DMFS between the two groups. This suggests that better locoregional control would result in better OS. Several dose escalation studies, using IMRT technique reported hopeful results. Ben-josef et al. conducted a phase I/II trial of IMRT dose escalation in LAPC patients [19]. They reported that high-dose radiation therapy, $55 \mathrm{~Gy} / 25 \mathrm{fr}$. can be administered safely with concurrent full-dose gemcitabine, and the median OS after such therapy was 15 months. Recently, Krishnan et al. reported that dose escalated IMRT (BED > $70 \mathrm{~Gy}$ ) for the patients who had tumors $>1-\mathrm{cm}$ from the luminal organs is feasible and tolerable [20]. They reported that higher dose (BED) was a strong independent predictor of improved OS in those patients. In our cohort, the patients who received over $45 \mathrm{~Gy}$, using IMRT had significantly better OS than those receiving 
Table 3 Univariate analysis of all patients

\begin{tabular}{|c|c|c|c|c|c|}
\hline Factors & $\begin{array}{l}\text { Number of } \\
\text { patients }\end{array}$ & $\begin{array}{l}\text { 1-year } \\
\text { LRPFS (\%) }\end{array}$ & $p$-value & $\begin{array}{l}\text { 1-year OS } \\
(\%)\end{array}$ & $p$-value \\
\hline \multicolumn{6}{|l|}{ Age (years) } \\
\hline$<66$ & 58 & 52.6 & 0.039 & 64.7 & 0.11 \\
\hline$\geq 66$ & 49 & 81.0 & & 85.5 & \\
\hline \multicolumn{6}{|l|}{ Gender } \\
\hline Male & 45 & 71.9 & 0.88 & 77.4 & 0.67 \\
\hline Female & 62 & 59.5 & & 72.0 & \\
\hline \multicolumn{6}{|l|}{ Tumor size } \\
\hline$<3 \mathrm{~cm}$ & 64 & 65.0 & 0.11 & 77.7 & 0.047 \\
\hline$\geq 3 \mathrm{~cm}$ & 43 & 66.7 & & 69.3 & \\
\hline \multicolumn{6}{|c|}{ Radiation modality } \\
\hline 3DCRT & 80 & 63.2 & 0.035 & 68.2 & 0.037 \\
\hline IMRT & 27 & 73.1 & & 92.3 & \\
\hline \multicolumn{6}{|l|}{ PS } \\
\hline 0 & 52 & 65.2 & 0.55 & 78.3 & 0.053 \\
\hline $1-2$ & 55 & 66.2 & & 70.5 & \\
\hline \multicolumn{6}{|l|}{$\begin{array}{l}\text { Pretreatment } \\
\text { CA19-9 (U/ml) }\end{array}$} \\
\hline$<300$ & 64 & 76.7 & 0.0026 & 82.2 & 0.010 \\
\hline$\geq 300$ & 43 & 50.0 & & 62.8 & \\
\hline \multicolumn{6}{|c|}{ Tumor location } \\
\hline Body/Tail & 50 & 69.4 & 0.24 & 76.0 & 0.21 \\
\hline Head/Neck & 57 & 62.3 & & 72.8 & \\
\hline
\end{tabular}

under 42 Gy of IMRT (MST 28.8 months vs. 14.3 months, $p<0.01$ ), which also suggests that dose escalation with IMRT for LAPC is a promising strategy.

As for recurrence pattern, patients who underwent IMRT had less locoregional recurrence as a first site compared with those who underwent 3DCRT (11.1\% vs. $30 \%)$. Locoregional relapse is often associated with pain,

Table 4 Multivariate analysis of all patients

\begin{tabular}{|c|c|c|c|}
\hline \multirow[t]{2}{*}{ Factors } & \multirow{2}{*}{$\begin{array}{l}\text { Number of } \\
\text { patients }\end{array}$} & \multicolumn{2}{|l|}{ OS } \\
\hline & & HR $(95 \% \mathrm{Cl})$ & $p$-value \\
\hline \multicolumn{4}{|c|}{ Radiation modality } \\
\hline 3DCRT & 80 & & \\
\hline IMRT & 27 & $0.64(0.38-1.06)$ & 0.082 \\
\hline \multicolumn{4}{|c|}{ Pretreatment CA19-9 (U/ml) } \\
\hline$<300$ & 64 & & \\
\hline$\geq 300$ & 43 & $1.62(1.06-2.47)$ & 0.026 \\
\hline \multicolumn{4}{|l|}{ Tumor size } \\
\hline$<3 \mathrm{~cm}$ & 64 & & \\
\hline$\geq 3 \mathrm{~cm}$ & 43 & $1.37(0.89-2.10)$ & 0.15 \\
\hline
\end{tabular}

Abbreviations; OS overall survival, $H R$ hazard ratio
Table 5 Univariate analysis of IMRT patients

\begin{tabular}{|c|c|c|c|c|c|}
\hline Factors & $\begin{array}{l}\text { Number of } \\
\text { patients }\end{array}$ & $\begin{array}{l}\text { 1-year } \\
\text { LRPFS (\%) }\end{array}$ & $p$-value & $\begin{array}{l}\text { 1-year } \\
\text { OS (\%) }\end{array}$ & $p$-value \\
\hline \multicolumn{6}{|l|}{ Age (years) } \\
\hline$<66$ & 12 & 50 & 0.015 & 83.3 & 0.21 \\
\hline$\geq 66$ & 15 & 92.9 & & 100 & \\
\hline \multicolumn{6}{|l|}{ Gender } \\
\hline Male & 17 & 68.8 & 0.63 & 93.8 & 0.72 \\
\hline Female & 10 & 80 & & 90 & \\
\hline \multicolumn{6}{|l|}{ Tumor size } \\
\hline$<3 \mathrm{~cm}$ & 19 & 72.2 & 0.36 & 94.4 & 0.23 \\
\hline$\geq 3 \mathrm{~cm}$ & 8 & 75 & & 87.5 & \\
\hline \multicolumn{6}{|l|}{ Radiation dose } \\
\hline$<45$ Gy & 9 & 33.3 & $1.23 \mathrm{E}-07$ & 88.9 & 0.000077 \\
\hline$\geq 45 \mathrm{~Gy}$ & 18 & 94.1 & & 94.1 & \\
\hline \multicolumn{6}{|l|}{ PS } \\
\hline 0 & 15 & 60 & 0.41 & 86.7 & 0.47 \\
\hline 1 & 12 & 90.9 & & 100 & \\
\hline \multicolumn{6}{|l|}{$\begin{array}{l}\text { Pretreatment } \\
\text { CA19-9 (U/ml) }\end{array}$} \\
\hline$<300$ & 17 & 87.5 & 0.032 & 100 & 0.037 \\
\hline$\geq 300$ & 10 & 50 & & 80 & \\
\hline \multicolumn{6}{|l|}{ Tumor location } \\
\hline Body/Tail & 11 & 54.5 & 0.25 & 81.8 & 0.12 \\
\hline Head/Neck & 16 & 86.7 & & 100 & \\
\hline
\end{tabular}

gastroduodenal obstruction. Therefore, improved locoregional control would be beneficial for keeping quality of life for the patients of LAPC. On the other hand, the rate of distant metastasis as a first site is still high which is approximately $50-60 \%$. This suggest that more efficient systemic chemotherapy is needed to treat micrometastatic spread in these patients. Low toxicity of CRT using IMRT will make it possible to receive further treatment with intensive systemic chemotherapies, and FOLFIRINOX or gemcitabine plus nab-paclitaxel regimens before or after CRT may address these issues better $[10,11]$.

As with any retrospective analysis, there are several limitations. First, the assessment of toxicities in a retrospective analysis tends to underestimate risks owing to incomplete recording of side effects and the recall bias. However, severe toxicities would have required additional medical care, which would have been clearly documented. Second, IMRT was administered as part of an institutional change in practice in 2009 , rather than in a prospective controlled manner. In addition, the dose of concurrent chemotherapy and the rate of patients who received induction chemotherapy were different between the 3DCRT and IMRT 

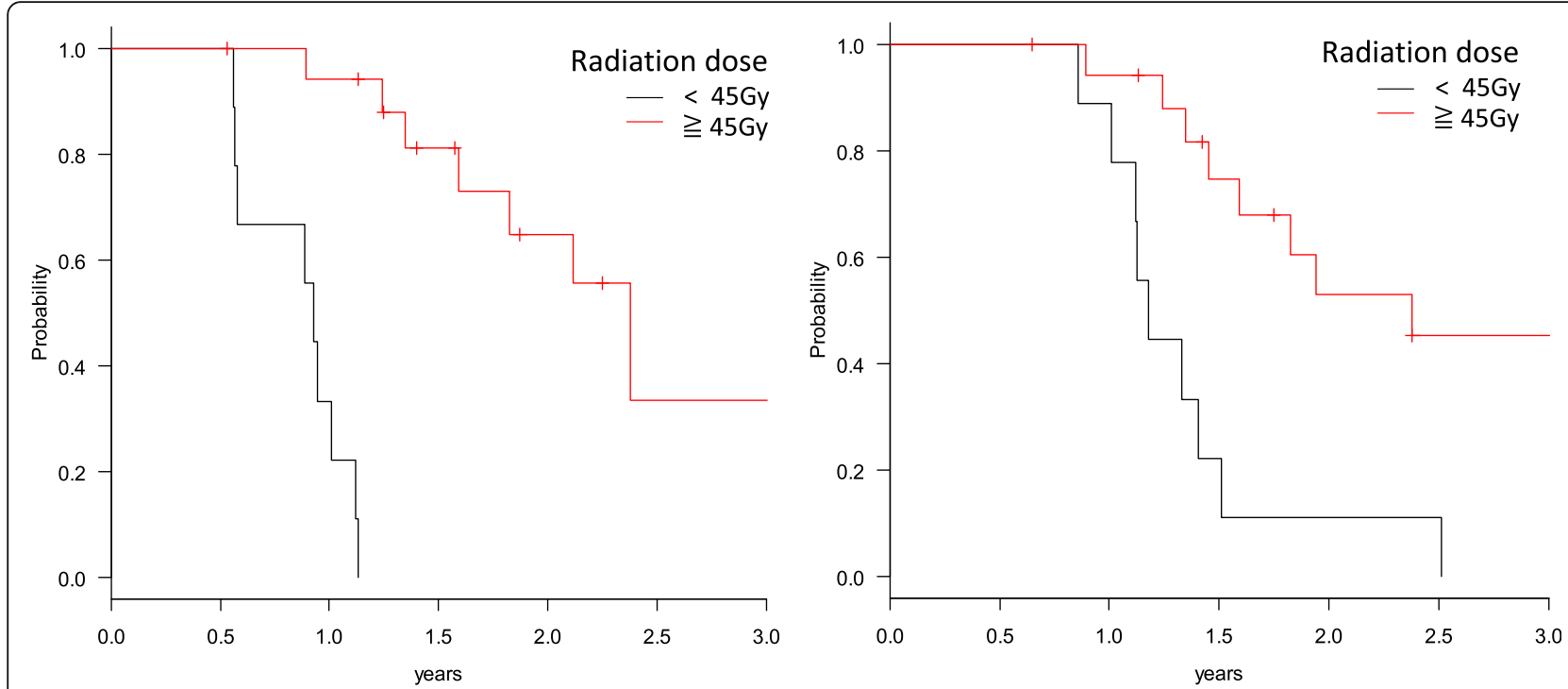

Fig. 2 Kaplan-Meier estimates of (a) Locoregional progression free survival (LRPFS) and (b) overall survival (OS), according to total dose $\geq 45$ Gy or $<45$ Gy

patients, and respiratory management was used in IMRT patients only. However, all treatment plans were developed and conducted at one institution, limiting the bias introduced by multi-institutional plans. Third, this retrospective analysis did not directly compare the outcomes between CRT and chemotherapy for LAPC patients. Clinical studies, including the LAP07 randomized clinical trial, which could not show survival benefit of CRT over chemotherapy alone for LAPC adopted the conventional radiotherapy technique, 3DCRT $[6,8]$. In this study, the MST of the patients treated with IMRT under 45 Gy was 14.3 months, which was approximately equal to the results of CRT or chemotherapy patients in LAP07 trial. On the other hand, the MST of the patients treated with IMRT $\geq 45$ Gy was 28.8 months, and it is much better than the result of the patients treated with IMRT under $45 \mathrm{~Gy}$. This result prompted us to verify the role of radiotherapy, especially IMRT, for LAPC patients.

In summary, our data demonstrated that LAPC patients treated with hypofractionated full-dose gemcitabine IMRT had improved OS and LRPFS without increased GI toxicities when compared to conventionally fractionated low dose gemcitabine 3DCRT, suggesting that intensified CRT, using IMRT might be beneficial for LAPC patients. To

Table 6 Multivariate analysis of IMRT patients

\begin{tabular}{|c|c|c|c|c|c|}
\hline \multirow[t]{2}{*}{ Factors } & \multirow{2}{*}{$\begin{array}{l}\text { Number of } \\
\text { patients }\end{array}$} & \multicolumn{2}{|l|}{ LRPFS } & \multicolumn{2}{|l|}{ OS } \\
\hline & & $\mathrm{HR}(95 \% \mathrm{Cl})$ & $p$-value & $\mathrm{HR}(95 \% \mathrm{Cl})$ & $p$-value \\
\hline \multicolumn{6}{|l|}{ Age (years) } \\
\hline$<66$ & 12 & & & & \\
\hline$\geq 66$ & 15 & $0.23(0.064-0.86)$ & 0.029 & $0.86(0.28-2.67)$ & 0.79 \\
\hline \multicolumn{6}{|l|}{ Radiation dose } \\
\hline$<45$ Gy & 9 & & & & \\
\hline$\geq 45$ Gy & 18 & $0.043(0.0049-0.38)$ & 4.00E-03 & $0.27(0.077-0.96)$ & 0.044 \\
\hline \multicolumn{6}{|c|}{ Pretreatment CA19-9 (U/ml) } \\
\hline$<300$ & 17 & & & & \\
\hline$\geq 300$ & 10 & $3.39(0.85-13.54)$ & 0.084 & $1.87(0.63-5.55)$ & 0.26 \\
\hline \multicolumn{6}{|l|}{ Tumor location } \\
\hline Body/Tail & 11 & & & & \\
\hline Head/Neck & 16 & $0.73(0.21-2.58)$ & 0.62 & $0.61(0.20-1.9)$ & 0.39 \\
\hline
\end{tabular}


Table 7 Acute and late gastrointestinal toxicity

\begin{tabular}{lllll}
\hline & Gr 0-1 & Gr 2 & Gr 3 & Gr 4 \\
\hline Acute Gl toxicity & & & & \\
3D-CRT & $48(60 \%)$ & $29(36 \%)$ & $3(4 \%)$ & $0(0 \%)$ \\
IMRT & $22(81 \%)$ & $5(19 \%)$ & $0(0 \%)$ & $0(0 \%)$ \\
Late GI toxicity & & & & \\
3DCRT & $70(88 \%)$ & $0(0 \%)$ & $10(12 \%)$ & $0(0 \%)$ \\
IMRT & $25(92 \%)$ & $1(4 \%)$ & $1(4 \%)$ & $0(0 \%)$ \\
\hline Abbreviations; Gl gastrointestinal & & &
\end{tabular}

evaluate this prospectively, we are currently conducting phase II multi-institutional clinical trial of CRT, using IMRT for LAPC patients (UMIN000017521).

\section{Conclusions}

LAPC patients treated with hypofractionated full-dose gemcitabine IMRT had improved OS and LRPFS without increased GI toxicities when compared to those of patients treated with conventionally fractionated low dose gemcitabine 3DCRT. In IMRT patients, higher dose was an independent favorable prognostic factor for better LRPFS and OS, which suggests that dose escalation with IMRT for LAPC is a promising strategy.

\section{Abbreviations \\ 3DCRT: three-dimensional conformal radiotherapy; BED: biological equivalent dose; CRT: chemoradiotherapy; CTCAE: Common Terminology Criteria for Adverse Events; CTV: clinical target volume; DMS: distant metastasis free survival; Gl: gastrointestinal; GTV: gross tumor volume; IMRT: intensity- modulated radiotherapy; LAPC: locally advanced pancreatic cancer; LRPFS: locoregional progression free survival; MST: median survival time; OS: overall survival; PTV: planned target volume}

\section{Funding}

This research was supported in part by the Practical Research for Innovative Cancer Control (17ck0106303h0001) from Japan Agency for Medical Research and Development (AMED). The funding program had no effect on the study design or the interpretation of data.

\section{Availability of data and materials}

The datasets used and/or analysed during the current study are available from the corresponding author on reasonable request.

\section{Authors' contributions}

YG participated in designing the study, data analysis, and writing the final manuscript. RA, KS, SM, MK, HI, TM, YK, KT, MH, and TM contributed to patient treatment, analysis, and review the manuscript. AN, SI, and KS contributed to data collection, patient treatment, analysis, and review the manuscript. All authors read and approved the final manuscript.

\section{Ethics approval and consent to participate}

All procedures performed in studies involving human participants were in accordance with the ethical standards of the institutional and/or national research committee and with the 1964 Helsinki Declaration and its later amendments or comparable ethical standards. Informed consent was obtained and chart reviews were performed after approval by the ethics committee of Kyoto University Hospital.

\section{Consent for publication}

Not Applicable.

\section{Competing interests}

The authors declare that they have no competing interests.

\section{Publisher's Note}

Springer Nature remains neutral with regard to jurisdictional claims in published maps and institutional affiliations.

\section{Author details}

${ }^{1}$ Department of Radiation Oncology and Image-applied Therapy, Kyoto University Graduate School of Medicine, 54 Shogoin, Kawaracho, Sakyo-ku, Kyoto, Japan. ${ }^{2}$ Department of Radiation Oncology, Graduate School of Medicine, Yamaguchi University, Yamaguchi, Japan. ${ }^{3}$ Department of Clinical Oncology, Kyoto University Graduate School of Medicine, Kyoto, Japan. ${ }^{4}$ Department of Diagnostic Imaging and Nuclear Medicine, Kyoto University Graduate School of Medicine, Kyoto, Japan. ${ }^{5}$ Department of Surgery, Kyoto University Graduate School of Medicine, Kyoto, Japan. ${ }^{6}$ Department of Gastroenterology and Hepatology, Kyoto University Graduate School of Medicine, Kyoto, Japan. ${ }^{7}$ Department of Radiation Oncology, Japanese Red Cross Society Wakayama Medical Center, Wakayama, Japan.

Received: 14 October 2017 Accepted: 14 June 2018

Published online: 25 June 2018

\section{References}

1. Willett CG, Czito BG, Bendell JC, Ryan DP. Locally advanced pancreatic cancer. Journal of clinical oncology : official journal of the American Society of Clinical Oncology. 2005:23:4538-44.

2. Krishnan $S$, Rana $V$, Janjan NA, Varadhachary GR, Abbruzzese $J L$, Das $P$, et al. Induction chemotherapy selects patients with locally advanced, unresectable pancreatic cancer for optimal benefit from consolidative chemoradiation therapy. Cancer. 2007;110:47-55.

3. Johung K, Saif MW, Chang BW. Treatment of locally advanced pancreatic cancer: the role of radiation therapy. Int J Radiat Oncol Biol Phys. 2012;82: 508-18.

4. lacobuzio-Donahue CA, Fu B, Yachida S, Luo M, Abe H, Henderson CM, et al. DPC4 gene status of the primary carcinoma correlates with patterns of failure in patients with pancreatic cancer. Journal of clinical oncology : official journal of the American Society of Clinical Oncology. 2009;27:1806-13.

5. Klaassen DJ, MacIntyre JM, Catton GE, Engstrom PF, Moertel CG. Treatment of locally unresectable cancer of the stomach and pancreas: a randomized comparison of 5-fluorouracil alone with radiation plus concurrent and maintenance 5-fluorouracil-an eastern cooperative oncology group study. J Clin Oncol : official journal of the American Society of Clinical Oncology. 1985;3:373-8

6. Chauffert B, Mornex F, Bonnetain F, Rougier P, Mariette C, Bouche $\mathrm{O}$, et al. Phase III trial comparing intensive induction chemoradiotherapy (60 Gy, infusional 5-FU and intermittent cisplatin) followed by maintenance gemcitabine with gemcitabine alone for locally advanced unresectable pancreatic cancer. Definitive results of the 2000-01 FFCD/SFRO study. Annals of oncology : official journal of the European society for. Med Oncol. 2008;19:1592-9.

7. Loehrer PJ Sr, Feng Y, Cardenes H, Wagner L, Brell JM, Cella D, et al. Gemcitabine alone versus gemcitabine plus radiotherapy in patients with locally advanced pancreatic cancer: an eastern cooperative oncology group trial. Journal of clinical oncology : official journal of the American Society of Clinical Oncology. 2011;29:4105-12.

8. Hammel P, Huguet F, van Laethem JL, Goldstein D, Glimelius B, Artru P, et al. Effect of Chemoradiotherapy vs chemotherapy on survival in patients with locally advanced pancreatic Cancer controlled after 4 months of gemcitabine with or without Erlotinib: the LAP07 randomized clinical trial. JAMA. 2016;315:1844-53.

9. Conroy T, Desseigne F, Ychou M, Bouche O, Guimbaud R, Becouarn Y, et al. FOLFIRINOX versus gemcitabine for metastatic pancreatic cancer. N Engl J Med. 2011;364:1817-25.

10. Suker M, Beumer BR, Sadot E, Marthey L, Faris JE, Mellon EA, et al. FOLFIRINOX for locally advanced pancreatic cancer: a systematic review and patient-level meta-analysis. The Lancet Oncology. 2016;17:801-10.

11. Von Hoff DD, Ervin T, Arena FP, Chiorean EG, Infante J, Moore M, et al. Increased survival in pancreatic cancer with nab-paclitaxel plus gemcitabine. N Engl J Med. 2013;369:1691-703.

12. Nakamura A, Shibuya K, Matsuo Y, Nakamura M, Shiinoki T, Mizowaki T, et al Analysis of dosimetric parameters associated with acute gastrointestinal toxicity and upper gastrointestinal bleeding in locally advanced pancreatic 
cancer patients treated with gemcitabine-based concurrent chemoradiotherapy. Int J Radiat Oncol Biol Phys. 2012;84:369-75.

13. Yovino S, Poppe M, Jabbour S, David V, Garofalo M, Pandya N, et al. Intensity-modulated radiation therapy significantly improves acute gastrointestinal toxicity in pancreatic and ampullary cancers. Int J Radiat Oncol Biol Phys. 2011;79:158-62.

14. Colbert LE, Moningi S, Chadha A, Amer A, Lee Y, Wolff RA, et al. Dose escalation with an IMRT technique in 15 to 28 fractions is better tolerated than standard doses of 3DCRT for LAPC. Advances in radiation oncology. 2017;2:403-15.

15. Oya N, Shibuya K, Sakamoto T, Mizowaki T. R, Fujimoto K, et al.

Chemoradiotherapy in patients with pancreatic carcinoma: phase-l study with a fixed radiation dose and escalating doses of weekly gemcitabine. Pancreatology : official journal of the international association of. Pancreatology. 2006:6:109-16.

16. Shibuya K, Oya N, Fujii T. R, Nakamura a, Matsuo Y, et al. phase II study of radiation therapy combined with weekly low-dose gemcitabine for locally advanced, unresectable pancreatic cancer. Am J Clin Oncol. 2011;34:115-9.

17. Murphy JD, Adusumilli S, Griffith KA, Ray ME, Zalupski MM, Lawrence TS, et al. Full-dose gemcitabine and concurrent radiotherapy for unresectable pancreatic cancer. Int J Radiat Oncol Biol Phys. 2007;68:801-8.

18. Bittner MI, Grosu AL, Brunner TB. Comparison of toxicity after IMRT and 3Dconformal radiotherapy for patients with pancreatic cancer - a systematic review. Radiotherapy and oncology : journal of the European Society for Therapeutic Radiology and Oncology. 2015;114:117-21.

19. Ben-Josef E, Schipper M, Francis IR, Hadley S, Ten-Haken R, Lawrence T, et al. A phase I/I trial of intensity modulated radiation (IMRT) dose escalation with concurrent fixed-dose rate gemcitabine (FDR-G) in patients with unresectable pancreatic cancer. Int J Radiat Oncol Biol Phys. 2012;84:1 166-71.

20. Krishnan S, Chadha AS, Suh Y, Chen HC, Rao A, Das P, et al. Focal radiation therapy dose escalation improves overall survival in locally advanced pancreatic Cancer patients receiving induction chemotherapy and consolidative Chemoradiation. Int J Radiat Oncol Biol Phys. 2016;94:755-65.

\section{Ready to submit your research? Choose BMC and benefit from}

- fast, convenient online submission

- thorough peer review by experienced researchers in your field

- rapid publication on acceptance

- support for research data, including large and complex data types

- gold Open Access which fosters wider collaboration and increased citations - maximum visibility for your research: over $100 \mathrm{M}$ website views per year

At BMC, research is always in progress.

Learn more biomedcentral.com/submissions 